\title{
Response of integrate-and-fire neurons to noisy inputs filtered by synapses with arbitrary timescales: firing rate and correlations Rubén Moreno-Bote*1,2 and Néstor Parga ${ }^{2}$
}

\author{
Address: ${ }^{1}$ Department of Brain and Cognitive Sciences, University of Rochester, Rochester, New York, 14627, USA and ${ }^{2}$ Dept. de Física Teórica. \\ Universidad Autónoma de Madrid, Cantoblanco 28049, Madrid, Spain \\ Email: Rubén Moreno-Bote* - rmoreno@bcs.rochester.edu \\ * Corresponding author
}

from Eighteenth Annual Computational Neuroscience Meeting: CNS*2009

Berlin, Germany. 18-23 July 2009

Published: 13 July 2009

BMC Neuroscience 2009, I0(Suppl I):P237 doi:I0.II86/I47|-2202-I0-SI-P237

This abstract is available from: http://www.biomedcentral.com//47/-2202//0/SI/P237

(c) 2009 Moreno-Bote and Parga; licensee BioMed Central Ltd.

\section{Introduction}

Delivery of neurotransmitter produces on a synapse a current that flows through the membrane and gets transmitted into the soma of the neuron, where it is integrated. The decay time of the current depends on the synaptic receptor's type, and ranges from a few (e.g. AMPA receptors) to a few hundred milliseconds (e.g. NMDA receptors). The role of the variety of synaptic timescales, several of them coexisting in the same neuron, is at present not understood. A prime question to answer is which is the effect of temporal filtering at different timescales of the incoming spike trains on the neuron's response.

\section{Results}

Here, based on our previous work on linear synaptic filtering [1-3], we build a general theory for the stationary firing response of integrate-and-fire (IF) neurons receiving stochastic inputs filtered by one, two or multiple synaptic channels each characterized by an arbitrary timescale. The formalism applies to arbitrary IF model neurons, and to arbitrary forms of input noise (i.e., not required to be Gaussian or to have small amplitude), as well as to any form of synaptic filtering (linear or non-linear). The theory determines with exact analytical expressions the firing rate of an IF neuron for long synaptic time constants using the adiabatic approach. The correlated spiking (cross-correlations function) of two neurons receiving common as well as independent sources of noise is also described (see figure 1). The theory is exemplified using leaky, quadratic and noise thresholded IF neurons (LIF, QIF, NTIF).
Although the adiabatic approach is exact when at least one of the synaptic timescales is long, it provides an excellent prediction of the firing rate even when the timescales of the synapses are comparable to that of the leak of the neuron; it is not required that the synaptic time constants are longer than the mean inter-spike-intervals. The distribution of the potentials for general IF neurons is also characterized.

\section{Conclusion}

Our results provide very powerful analytical tools that can allow a quantitative description of the dynamics of neuronal networks with realistic synaptic dynamics.

\section{References}

I. Moreno-Bote R, Parga N: Simple model neurons with slow synaptic filters can operate as detectors of rare events. Physical Review Letters 2004, 92:028I02.

2. Moreno-Bote R, Parga N: Membrane potential and response properties of populations of cortical neurons in the high conductance state. Physical Review Letters 2005, 94:088I03.

3. Moreno-Bote R, Parga N: Auto and cross-correlograms for the spike response of LIF neurons with slow synapses. Physical Review Letters 2006, 96:02810I. 
A

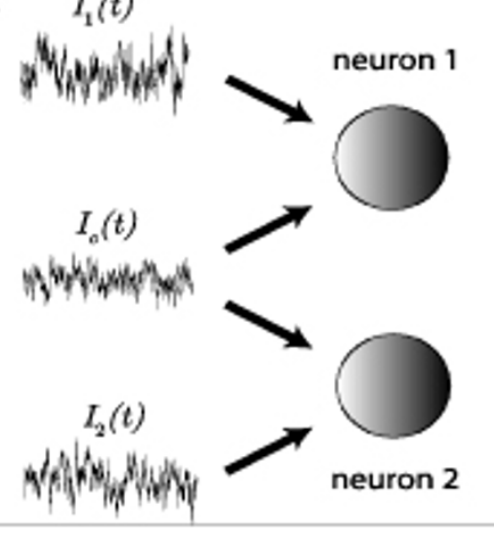

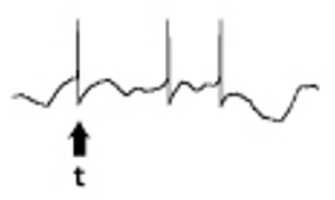

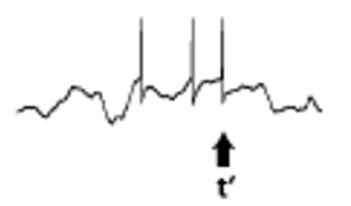

B

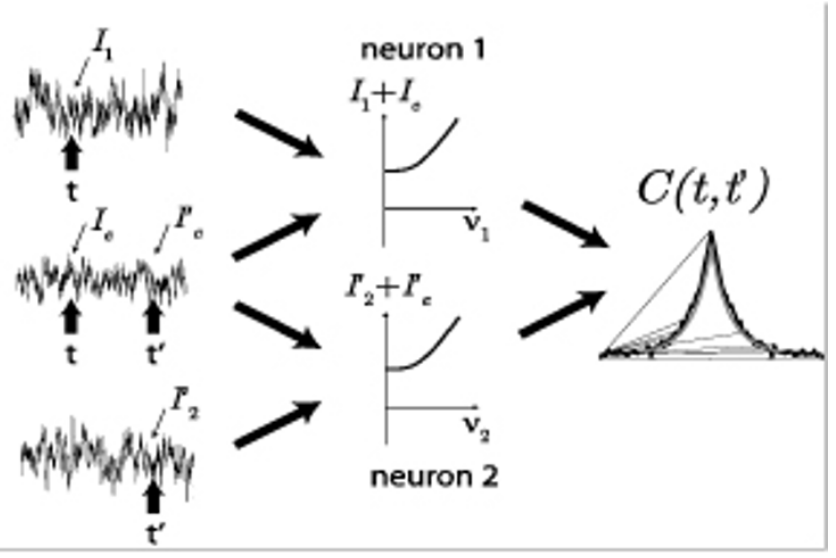

Figure I

A. Two spiking neurons receiving correlated/common inputs fire in a correlated manner. B: The cross-correlated output spike trains of the two neurons are analytically described.

Publish with Bio Med Central and every scientist can read your work free of charge

"BioMed Central will be the most significant development for disseminating the results of biomedical research in our lifetime. "

Sir Paul Nurse, Cancer Research UK

Your research papers will be:

- available free of charge to the entire biomedical community

- peer reviewed and published immediately upon acceptance

- cited in PubMed and archived on PubMed Central

- yours - you keep the copyright 